\title{
RESEARCH
}

Open Access

\section{Research diagnostic criteria for Alzheimer's disease: findings from the LipiDiDiet randomized controlled trial}

\author{
Anna Rosenberg ${ }^{1 *+} \mathbb{D}$, Alina Solomon ${ }^{1,2,3 \dagger}$, Hilkka Soininen ${ }^{1,4}$, Pieter Jelle Visser ${ }^{5,6}$, Kaj Blennow ${ }^{7,8}$, \\ Tobias Hartmann ${ }^{9}$, Miia Kivipelto $1,2,3,10,11$ and on behalf of the LipiDiDiet clinical study group
}

\begin{abstract}
Background: To explore the utility of the International Working Group (IWG)-1 criteria in recruitment for Alzheimer's disease (AD) clinical trials, we applied the more recently proposed research diagnostic criteria to individuals enrolled in a randomized controlled prevention trial (RCT) and assessed their disease progression.
\end{abstract}

Methods: The multinational LipiDiDiet RCT targeted 311 individuals with IWG-1 defined prodromal AD. Based on centrally analyzed baseline biomarkers, participants were classified according to the IWG-2 and National Institute on Aging-Alzheimer's Association (NIA-AA) 2011 and 2018 criteria. Linear mixed models were used to investigate the 2-year change in cognitive and functional performance (Neuropsychological Test Battery NTB Z scores, Clinical Dementia Rating-Sum of Boxes CDR-SB) (criteria $\times$ time interactions; baseline score, randomization group, sex, MiniMental State Examination (MMSE), and age also included in the models). Cox models adjusted for randomization group, MMSE, sex, age, and study site were used to investigate the risk of progression to dementia over 2 years.

Results: In total, 88\%, 86\%, and 69\% of participants had abnormal cerebrospinal fluid (CSF) $\beta$-amyloid, total tau, and phosphorylated tau, respectively; $64 \%$ had an $\mathrm{A}+\mathrm{T}+\mathrm{N}+$ profile (CSF available for $N=107$ ). Cognitive-functional decline appeared to be more pronounced in the IWG-2 prodromal AD, NIA-AA 2011 high and intermediate AD likelihood, and NIA-AA 2018 AD groups, but few significant differences were observed between the groups within each set of criteria. Hazard ratio (95\% Cl) for dementia was 4.6 (1.6-13.7) for IWG-2 prodromal AD (reference group no prodromal AD), 7.4 (1.0-54.7) for NIA-AA 2011 high AD likelihood (reference group suspected non-AD pathology SNAP), and 9.4 (1.2-72.7) for NIA-AA 2018 AD (reference group non-Alzheimer's pathologic change). Compared with the NIA-AA 2011 high AD likelihood group (abnormal $\beta$-amyloid and neuronal injury markers), disease progression was similar in the intermediate AD likelihood group (medial temporal lobe atrophy; no CSF available).

Conclusions: Despite being less restrictive than the other criteria, the IWG-1 criteria reliably identified individuals with AD pathology. More pragmatic and easily applicable selection criteria might be preferred due to feasibility in certain situations, e.g., in multidomain prevention trials that do not specifically target $\beta$-amyloid/tau pathologies.

\footnotetext{
* Correspondence: anna.rosenberg@uef.fi

${ }^{\dagger}$ Anna Rosenberg and Alina Solomon contributed equally to this work. ${ }^{1}$ Department of Neurology, Institute of Clinical Medicine, University of Eastern Finland, Kuopio, Finland

Full list of author information is available at the end of the article
}

(c) The Author(s). 2021 Open Access This article is licensed under a Creative Commons Attribution 4.0 International License, which permits use, sharing, adaptation, distribution and reproduction in any medium or format, as long as you give appropriate credit to the original author(s) and the source, provide a link to the Creative Commons licence, and indicate if changes were made. The images or other third party material in this article are included in the article's Creative Commons. licence, unless indicated otherwise in a credit line to the material. If material is not included in the article's Creative Commons licence and your intended use is not permitted by statutory regulation or exceeds the permitted use, you will need to obtain permission directly from the copyright holder. To view a copy of this licence, visit http://creativecommons.org/licenses/by/4.0/ The Creative Commons Public Domain Dedication waiver (http://creativecommons.org/publicdomain/zero/1.0/) applies to the data made available in this article, unless otherwise stated in a credit line to the data. 
(Continued from previous page)

Trial registration: Netherlands Trial Register, NL1620. Registered on 9 March 2009

Keywords: Alzheimer's disease, Prodromal Alzheimer's disease, Randomized controlled trial, Prevention, Disease progression, Research criteria, Early diagnosis, Biomarkers, Cerebrospinal fluid

\section{Background}

To decelerate cognitive decline and delay dementia onset in Alzheimer's disease (AD), preventive interventions may need to target high-risk individuals early in the disease course. Several research diagnostic criteria relying on biomarker evidence for $\beta$-amyloid $(A \beta)$ and neuronal injury have been proposed to identify such individuals for randomized controlled trials (RCTs) [1-7]. Compared to biomarker-positive individuals, more heterogeneous groups of individuals and those without confirmed AD pathology may have a lower likelihood of cognitive decline [8]. A lower than expected decline in the placebo group combined with a lower than expected effect in the intervention group could mask beneficial treatment effects in RCTs. Indeed, past RCT failures might partly be attributable to suboptimal participant selection $[9,10]$.

The International Working Group (IWG)-1 research criteria for $\mathrm{AD}$ [1] attribute equal weight to all biomarkers, whereas the newer criteria emphasize A $\beta$ [3-7]. Most ongoing prodromal AD RCTs also require $A \beta$ positivity [11]. Biomarker-focused selection criteria and their impact on trial design have been investigated in simulation studies [11-16], but little is known about the operationalization of these criteria in real-life RCTs. Given that the more recently proposed criteria require comprehensive biomarker assessments, it is also relevant to study the potential utility of IWG-1 and other more easily applicable criteria in the recruitment for prevention RCTs. The multinational LipiDiDiet RCT, which investigated the effects of medical food on cognition in prodromal AD, was one of the first RCTs using the IWG-1 criteria in participant selection [17]. To understand whether these criteria can be successfully applied to identify individuals with $\mathrm{AD}$ biomarker profiles, we investigated LipiDiDiet participants' baseline biomarker profiles and classified them according to all other currently available research criteria: the IWG-2, National Institute on Aging-Alzheimer's Association (NIA-AA) 2011, and NIA-AA 2018 criteria. Furthermore, to understand the impact of biomarker profile on disease progression, we assessed the 2-year change in cognitive and functional performance as well as progression to dementia in the groups within each set of criteria.

\section{Methods}

\section{Trial design and participants}

LipiDiDiet is a double-blind proof-of-concept RCT conducted at 11 sites in Finland, Sweden, Germany, and the
Netherlands [17]. The 2-year core trial, completed in 2015, was followed by up to four 1-year double-blind extension studies. LipiDiDiet included 311 individuals aged $55-85$ years, recruited mainly from memory clinics. For eligibility, participants were required to meet the IWG-1 criteria for prodromal $\mathrm{AD}$ [1], i.e., have an underlying AD pathology and mild episodic memory impairment, defined in LipiDiDiet as a performance below one standard deviation in at least two cognitive tests (at least one memory test). The following cognitive screening tests were used: Free and Cued Selective reminding test, free and delayed recall, Wechsler Memory Scale-revised (WMS-r) story, delayed recall, WMS-r delayed recall of figures, Trail Making Tests A and B, symbol digit substitution test, and category fluency test. Sufficient evidence for $\mathrm{AD}$ pathology was defined as abnormality in at least one of the following biomarkers: cerebrospinal fluid (CSF) $A \beta$ (A 342 and/or A $342 / 40$ ratio), CSF total tau (ttau), CSF phosphorylated tau (p-tau), fluoro-deoxyglucose-positron emission tomography (FDG-PET), or medial temporal lobe atrophy (MTA) on magnetic resonance imaging (MRI). Due to feasibility, MTA was often the primary biomarker used to assess eligibility in LipiDiDiet.

Exclusion criteria were dementia diagnosis or substantial cognitive impairment (Mini-Mental State Examination (MMSE) $<24$ or $<20$ if $\leq 6$ years of education), cholinesterase inhibitor or memantine use, neuroimaging abnormalities (stroke, intracranial bleeding, mass lesion, normal pressure hydrocephalus), and conditions potentially interfering with participation (e.g., alcohol/ drug abuse). Individuals taking omega-3 products or vitamin B6, B12, C, E, or folic acid ( $>200 \%$ of the recommended daily intake) were also excluded due to the nature of the intervention.

LipiDiDiet is registered at Netherlands Trial Register (identifier NL1620). Ethical approval was granted by local ethics committees, and written informed consent was obtained from all participants and study partners prior to enrollment.

\section{Trial protocol}

Participants were randomized in a 1:1 ratio to the intervention and control groups. The intervention group consumed once a day a 125-ml medical food drink (Souvenaid ${ }^{\circledR}$ ) containing the multinutrient Fortasyn Connect $^{\mathrm{TM}}$ (Nutricia; Zoetermeer, the Netherlands). Fortasyn 
Connect $^{\text {ta }}$ consists of a combination of nutrients which AD patients might be deficient in [18], including omega3 fatty acids, vitamins, folic acid, phospholipids, and antioxidants. In short-term RCTs in mild AD, this multinutrient showed beneficial effects on cognitive performance and brain functional connectivity $[19,20]$. The control group consumed once a day an iso-caloric placebo product similar in appearance, flavor, and composition, but without the multinutrient. The participants, clinical team, and outcome evaluators were blinded to group assignment. Main study parameters and outcomes were assessed at baseline, 6,12 , and 24 months. For motivation and compliance, additional visits with the study nurse or physician were organized at 3,9 , and 18 months, and phone calls were conducted monthly for the first 6 months and every 2 months after that. Protocol details are described elsewhere [17].

\section{Cognitive outcomes and progression to dementia}

The primary trial outcome was change in cognitive performance measured with a Neuropsychological Test Battery (NTB) composite $Z$ score including five tests: Consortium to Establish a Registry for Alzheimer's Disease (CERAD) 10-word list learning, delayed recall, and recognition, category fluency test, and letter digit substitution test. Secondary cognitive outcomes included the domain-specific $Z$ scores for memory (three tests; CERAD 10-word list learning, delayed recall, and recognition) and executive functioning (four tests; category fluency test, letter digit substitution test, concept shifting test condition $C$, and WMS-r digit span), as well as the NTB total $Z$ score. The total score was based on 16 tests (the above-mentioned tests and additionally WMS-r logical verbal memory, immediate and delayed recall, WMS-r visual paired associates, immediate and delayed recall, 30-item Boston Naming Test, CERAD constructional praxis, copy and recall, and concept shifting test conditions A and B). Test scores were calculated as standardized $Z$ scores with higher scores demonstrating better performance. Cognition was assessed by study psychologists at baseline, 6,12 , and 24 months.

Other secondary outcomes, including the Clinical Dementia Rating-Sum of Boxes (CDR-SB) score reflecting global cognitive-functional performance, were assessed at baseline, 12, and 24 months. Dementia and AD were diagnosed using the Diagnostic and Statistical Manual of Mental Disorders 4th edition (DSM-IV) [21] and the National Institute of Neurological and Communicative Disorders and Stroke-Alzheimer's Disease and Related Disorders Association (NINCDS-ADRDA) criteria [22].

\section{Biomarker assessments}

A harmonized and optimized protocol for CSF sampling was followed at all sites. Lumbar puncture was performed in the morning and $10 \mathrm{ml}$ CSF was tapped by gravity drip. Samples were centrifuged $(2000 g, 10 \mathrm{~min},+$ $4{ }^{\circ} \mathrm{C}$ ), aliquoted in polypropylene tubes, and stored at $70-80^{\circ} \mathrm{C}$ for centralized analysis. Samples were first analyzed locally or sent for central laboratory analysis to assess eligibility at screening (pre-specified cut-offs for abnormality adjusted if necessary). For the evaluation of biomarker profiles and research diagnostic criteria, all samples, including those originally obtained for local analysis only, were analyzed or re-analyzed centrally in the Clinical Neurochemistry Laboratory at Sahlgrenska University Hospital, Mölndal, Sweden. Samples were handled by board-certified laboratory technicians and analyzed simultaneously using the same reagent batch, adhering to strict procedures for run acceptance and quality control procedures. $A \beta 40$ and $A \beta 42$ concentrations were measured using the MSD Abeta Triplex (Meso Scale Discovery, Rockville, Maryland) and the $\mathrm{A} \beta 42 / 40$ ratio was calculated as $(\mathrm{A} \beta 42 / \mathrm{A} \beta 40) \times 10$. $\mathrm{T}$ tau and p-tau were measured using INNOTEST sandwich ELISAs (Fujirebio, Ghent, Belgium). The following cut-offs for CSF abnormality were applied in the LipiDiDiet central analysis and in the present study: $\mathrm{A} \beta 42<$ $450 \mathrm{pg} / \mathrm{ml} ; \mathrm{A} \beta 42 / 40$ ratio $<1 ; \mathrm{t}$-tau $>350 \mathrm{pg} / \mathrm{ml} ; \mathrm{p}$-tau $>$ $60 \mathrm{pg} / \mathrm{ml}$.

At each study site, structural MRI was performed according to local scanning protocols. To assess MTA, coronal reconstructions of 3D T1-weighted scans were visually rated according to the Scheltens scale ranging from 0 (no atrophy) to 4 (severe atrophy). The right and left hemispheres were rated separately, and the sum was calculated. Scans were assessed locally to determine eligibility at screening. For the present study, results of the centralized analysis were used to assess biomarker profiles. Central analysis was conducted at the VU University Medical Center in Amsterdam, the Netherlands. MTA was defined as a score of $\geq 1$.

\section{Classification according to the research diagnostic criteria} In the present study, all participants (both the intervention and control group participants) with centrally analyzed baseline biomarkers (CSF and/or MTA; $N=287$ ) were classified according to the A/T/N biomarker scheme [23] and the IWG-2 [5], NIA-AA 2011 [3], and NIA-AA 2018 [7] research diagnostic criteria (Table 1). Biomarkers considered in the classification were MTA (imaging marker for neuronal injury) and CSF biomarkers reflecting $A \beta$ deposition ( $A \beta 42, A \beta 42 / 40$ ratio) and neuronal injury ( $p$-tau, $\mathrm{t}$-tau). $\mathrm{A} \beta$ or tau positron emission tomography (PET) scans were not performed in LipiDiDiet.

Participants were considered to have prodromal AD (IWG-2) if they had abnormal $A \beta$ and at least one abnormal CSF neuronal injury marker. High AD likelihood (NIA-AA 2011) was defined as abnormal $A \beta$ and at least 
Table 1 Biomarker profiles and classification according to the research diagnostic criteria for AD

\begin{tabular}{|c|c|}
\hline Criteria & Biomarker profile \\
\hline \multicolumn{2}{|l|}{ IWG-1-trial eligibility criteria } \\
\hline No prodromal AD & Normal CSF A $\beta$, t-tau, p-tau, MTA, FDG-PET \\
\hline Prodromal AD & Abnormal CSF A $\beta$, t-tau, p-tau, MTA, and/or FDG-PET \\
\hline \multicolumn{2}{|l|}{ IWG-2 } \\
\hline No prodromal AD & Normal CSF A $\beta+$ normal/abnormal CSF t-tau, p-tau, MTA \\
\hline Prodromal AD & Abnormal CSF A $\beta+$ abnormal CSF t-tau and/or p-tau \\
\hline \multicolumn{2}{|l|}{ NIA-AA 2011} \\
\hline Low AD likelihood & Normal CSF A $\beta+$ normal CSF t-tau, p-tau, and MTA \\
\hline Isolate amyloid pathology & Abnormal CSF A $\beta+$ normal CSF t-tau, p-tau, and MTA \\
\hline Suspected non-AD pathology (SNAP) & Normal CSF A $\beta+$ abnormal CSF t-tau, p-tau, and/or MTA \\
\hline High AD likelihood & Abnormal CSF A $\beta+$ abnormal CSF t-tau, p-tau, and/or MTA \\
\hline Intermediate AD likelihood & CSF biomarkers not available, abnormal MTA \\
\hline Inconclusive/uninformative & CSF biomarkers not available, normal MTA \\
\hline \multicolumn{2}{|l|}{ NIA-AA 2018} \\
\hline Normal AD biomarkers (A-T-N-) & Normal CSF A $\beta+$ normal CSF t-tau, p-tau, and MTA \\
\hline Alzheimer's pathologic change $(\mathrm{A}+\mathrm{T}-\mathrm{N}-)$ & Abnormal CSF A $\beta+$ normal CSF t-tau, p-tau, and MTA \\
\hline Non-Alzheimer's pathologic change (A-T+N, $\pm A-T-N+)$ & Normal CSF A $\beta+$ abnormal CSF p-tau, t-tau, and/or MTA \\
\hline $\begin{array}{l}\text { Alzheimer's and concomitant suspected non-Alzheimer's } \\
\text { pathologic change }(\mathrm{A}+\mathrm{T}-\mathrm{N}+)\end{array}$ & $\begin{array}{l}\text { Abnormal CSF A } \beta+\text { normal CSF p-tau + abnormal CSF t-tau } \\
\text { and/or MTA }\end{array}$ \\
\hline $\mathrm{AD}(\mathrm{A}+\mathrm{T}+\mathrm{N} \pm)$ & $\begin{array}{l}\text { Abnormal CSF A } \beta+\text { abnormal CSF p-tau + normal/abnormal } \\
\text { CSF t-tau and/or MTA }\end{array}$ \\
\hline
\end{tabular}

Abnormal CSF $A \beta$ defined as $A \beta 42<450 \mathrm{pg} / \mathrm{ml}$ and $/$ or $A \beta 42 / 40 \times 10<1$; abnormal CSF t-tau $>350 \mathrm{pg} / \mathrm{ml}$; abnormal CSF p-tau $>60$ pg $/ \mathrm{ml}$; abnormal MTA visual rating $\geq 1$

Abbreviations: $A \beta$, $\beta$-amyloid; $A D$, Alzheimer's disease; CSF, cerebrospinal fluid; FDG-PET, fluoro-deoxy-glucose-positron emission tomography; IWG, International Working Group; MTA, medial temporal lobe atrophy, visual rating; NIA-AA, National Institute on Aging-Alzheimer's Association; $p$-tau, phosphorylated tau at threonine 181 ; SNAP, suspected non-AD pathology; $t$-tau, total tau

one (any) abnormal neuronal injury marker. Participants with conflicting biomarkers were classified either in the isolate amyloid pathology group (abnormal $\mathrm{A} \beta$ but normal neuronal injury markers) or in the suspected non$\mathrm{AD}$ pathology (SNAP) group (normal $\mathrm{A} \beta$ but at least one abnormal neuronal injury marker) [24]. Participants with only one biomarker available (in LipiDiDiet MTA) were classified as having either an intermediate AD likelihood or an inconclusive/uninformative status, depending on whether MTA was present. According to the NIA-AA 2018 criteria, participants with abnormal A $\beta$ and $\mathrm{p}$-tau were considered to have $\mathrm{AD}(\mathrm{A}+\mathrm{T}+\mathrm{N} \pm$ profile). Participants with abnormal $A \beta$ and neuronal injury markers but normal p-tau were classified in the Alzheimer's and concomitant suspected non-Alzheimer's pathologic change group $(\mathrm{A}+\mathrm{T}-\mathrm{N}+)$. In case of conflicting biomarkers, participants were classified as having an Alzheimer's $(\mathrm{A}+\mathrm{T}-\mathrm{N}-)$ or non-Alzheimer's pathologic change $(\mathrm{A}-\mathrm{T}+\mathrm{N} \pm, \mathrm{A}-\mathrm{T}-\mathrm{N}+)$.

\section{Statistical analysis}

Between-group differences in participant baseline characteristics were analyzed with $t$-tests and chi-square tests, as appropriate. $Z$ scores for NTB composite, total, memory, and executive functioning were calculated as previously described [17]. We analyzed change from baseline in the NTB and CDR-SB scores using linear mixed models for repeated measures as previously reported, with baseline score, randomized treatment, time, treatment $\times$ time interaction, and baseline MMSE as fixed effects [17]. We additionally included the biomarker profile (research criteria) and criteria $\times$ time interaction, as well as sex and baseline age, as fixed effects in the present study. All trial participants regardless of randomized treatment were included in the analyses (intervention effects were accounted for by including randomized treatment and treatment $\times$ time interaction in the models). A random intercept with a variance components covariance structure was used within sites and a random intercept and slope for time with an unstructured covariance structure within subjects. Least-squares means for change from baseline were estimated from the linear mixed model. $p$ values are shown for the difference in least-squares means over 2 years between each group and the respective reference group. 
Associations between the biomarker profile (research criteria) and 2-year risk of progression to dementia were analyzed with Cox proportional hazards models adjusted for randomized treatment (to account for intervention effects), baseline MMSE, sex, baseline age, and study site. Results are presented as hazard ratios (HR) and 95\% CI. Results of all longitudinal analyses are reported for the modified intention-to-treat (mITT) population (all randomized participants with at least one post-baseline assessment, excluding visit data after progression to dementia and start of $\mathrm{AD}$ medication and/or open-label Souvenaid). SAS software version 9.4 was used in the analyses, and level of statistical significance was $<0.05$. All analyses were post hoc.

\section{Results}

Baseline characteristics and classification according to the research diagnostic criteria

Baseline characteristics of the participants with centrally assessed biomarkers (MTA on MRI and/or CSF) are shown in Table 2 . Out of 287 participants with at least one centrally analyzed baseline biomarker, 180 (62.7\%) had only MTA assessment available; CSF was analyzed for 107 participants (37.3\%). Participants with CSF available were younger and had lower CDRSB. In total, $62.3 \%$ of the participants with available apolipoprotein $\mathrm{E}(A P O E)$ data were $\varepsilon 4$ carriers. MTA score was at least 1 in $86.4 \%$ (241 out of 279 ) of the participants. CSF A $\beta$, t-tau, and p-tau levels were abnormal in $87.9 \%$ (94 out of 107), 86.0\% (92 out of 107 ), and $69.2 \%$ (74 out of 107) of the participants, respectively. Classification according to the $\mathrm{A} / \mathrm{T} / \mathrm{N}$ biomarker scheme is shown in Fig. 1. The majority, $63.6 \%$, had an $\mathrm{A}+\mathrm{T}+\mathrm{N}+$ profile.

Figure 2 illustrates the classification according to the different research diagnostic criteria. In total, 75.7\% (81 out of 107) of the participants had IWG-2 prodromal AD. Approximately half of the participants were classified in the NIA-AA 2011 intermediate AD likelihood group $(54.0 \%, 155$ out of 287 ) and $8.7 \%$ (25 out of 287 ) in the inconclusive/uninformative group. A third had a

Table 2 Baseline characteristics of the study population

\begin{tabular}{|c|c|c|c|c|c|}
\hline Characteristics & $N$ data available & $\begin{array}{l}\text { CSF and/or MRI } \\
\text { available }(N=287)\end{array}$ & $\begin{array}{l}\text { Only MRI available } \\
(N=180)\end{array}$ & $\begin{array}{l}\text { CSF available } \\
(N=107)\end{array}$ & $p$-value \\
\hline \multicolumn{6}{|l|}{ Demographics } \\
\hline Age, years & 287 & $70.9(6.6)$ & $71.6(6.2)$ & $69.8(7.0)$ & 0.02 \\
\hline Female & 287 & $147(51.2 \%)$ & $100(55.6 \%)$ & $47(43.9 \%)$ & 0.06 \\
\hline Education, years & 287 & $10.5(3.7)$ & $10.6(3.8)$ & $10.4(3.7)$ & 0.62 \\
\hline \multicolumn{6}{|l|}{ Cognition } \\
\hline MMSE score & 286 & $26.6(2.0)$ & $26.7(2.0)$ & $26.6(1.9)$ & 0.94 \\
\hline NTB composite & 284 & $0.012(0.682)$ & $-0.010(0.711)$ & $0.050(0.630)$ & 0.48 \\
\hline NTB memory & 283 & $0.014(0.848)$ & $0.021(0.885)$ & $0.003(0.786)$ & 0.87 \\
\hline NTB executive functioning & 281 & $0.007(0.681)$ & $-0.046(0.656)$ & $0.095(0.718)$ & 0.09 \\
\hline NTB total & 283 & $0.004(0.549)$ & $-0.003(0.561)$ & $0.017(0.532)$ & 0.77 \\
\hline CDR-SB & 259 & $1.76(1.12)$ & $1.91(1.17)$ & $1.53(1.02)$ & 0.01 \\
\hline \multicolumn{6}{|l|}{ Biomarkers and $A P O E$} \\
\hline APOE ع4 carrier & 260 & $162 / 260(62.3 \%)$ & $97 / 156(62.2 \%)$ & $65 / 104(62.5 \%)$ & 0.96 \\
\hline MTA score & 279 & $2[1-4]$ & $3[1-4]$ & $2[1-3]$ & 0.11 \\
\hline Abnormal MTA & 279 & $241 / 279(86.4 \%)$ & 155/180 (86.1\%) & 86/99 (86.9\%) & 0.86 \\
\hline CSF $A \beta 42, \mathrm{pg} / \mathrm{ml}$ & 107 & $412(242)$ & NA & $412(242)$ & NA \\
\hline CSF A $\beta 42 / 40$ ratio & 107 & $0.63(0.27)$ & NA & $0.63(0.27)$ & NA \\
\hline Abnormal $A \beta$ & 107 & 94/107 (87.9\%) & NA & $94 / 107$ (87.9\%) & NA \\
\hline CSF t-tau, pg/ml & 107 & $616(276)$ & NA & $616(276)$ & NA \\
\hline Abnormal t-tau & 107 & 92/107 (86.0\%) & NA & 92/107 (86.0\%) & NA \\
\hline CSF p-tau, pg/ml & 107 & 78 (29) & NA & 78 (29) & NA \\
\hline Abnormal p-tau & 107 & 74/107 (69.2\%) & NA & 74/107 (69.2\%) & NA \\
\hline
\end{tabular}

Data are mean (SD), median [IQR], or $N$ (\%). $p$-values are shown for comparisons between participants with only MRI available and those with centrally analyzed CSF. Abnormal MTA defined as a score of $\geq 1$; abnormal $A \beta$ as CSF A $342<450 \mathrm{pg} / \mathrm{ml}$ and/or CSF A $42 / 40$ ratio $<1$; abnormal t-tau as CSF t-tau $>350 \mathrm{pg} / \mathrm{ml}$; abnormal p-tau as CSF p-tau $>60 \mathrm{pg} / \mathrm{ml}$

Abbreviations: $A \beta 42, \beta$-amyloid 1-42; $A \beta 40, \beta$-amyloid 1-40; $A \beta 42 / 40$ ratio, A $\beta 42 / 40 \times 10 ; A P O E$, apolipoprotein E; CDR-SB, Clinical Dementia Rating-Sum of Boxes; $C S F$, cerebrospinal fluid; MMSE, Mini-Mental State Examination; MRI, magnetic resonance imaging; MTA, medial temporal lobe atrophy, visual rating; NTB, Neuropsychological Test Battery; p-tau, tau phosphorylated at threonine 181; $t$-tau, total tau 


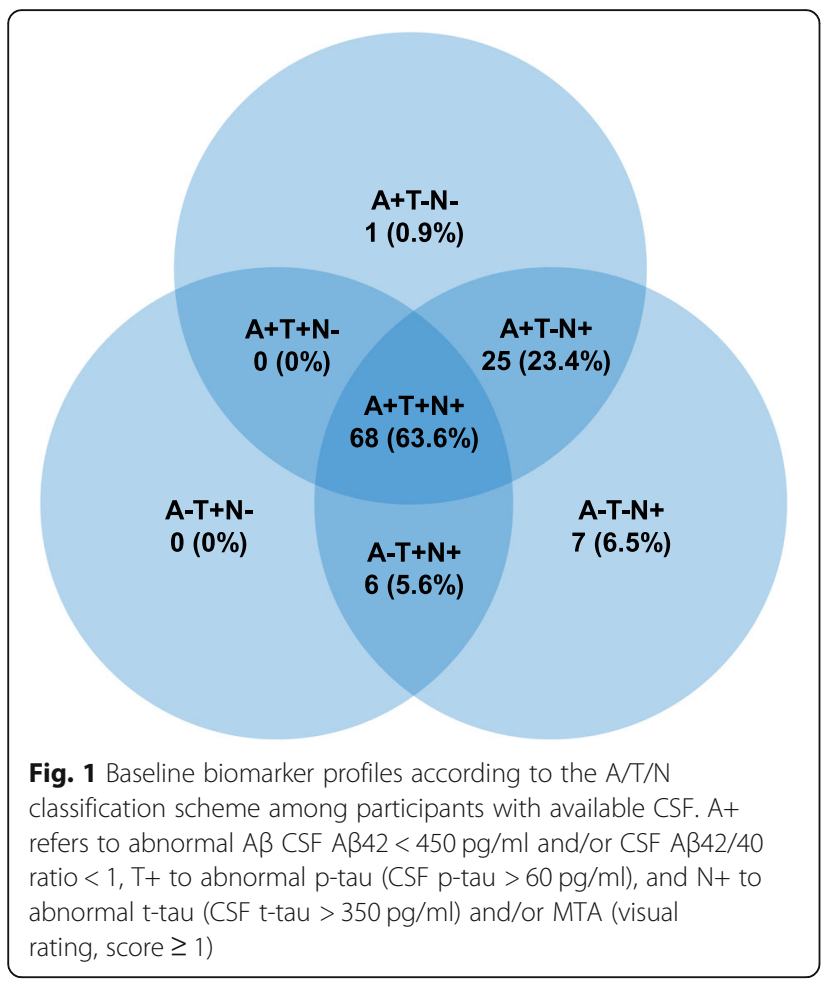

high $\mathrm{AD}$ likelihood (32.4\%, 93 out of 287 ) and 4.5\% (13 out of 287) were categorized in the SNAP group. One had an isolate amyloid pathology; no one had a low AD likelihood. With respect to the NIA-AA 2018 criteria, $63.6 \%$ of the participants (68 out of 107) were classified in the AD group, $23.4 \%$ (25 out of 107) in the Alzheimer's and concomitant suspected non-Alzheimer's pathologic change group, and $12.1 \%$ (13 out of 107) in the non-Alzheimer's pathologic change group. One had an Alzheimer's pathologic change. However, even a slight adjustment of the p-tau cut-off ( $>55 \mathrm{pg} / \mathrm{ml}$ instead of $>60 \mathrm{pg} / \mathrm{ml}$ ) changed the classification: in this case, more participants were assigned to the AD group (72.0\%, 77 out of 107) and fewer participants to the Alzheimer's and concomitant suspected non-Alzheimer's pathologic change group (15.0\%, 16 out of 107). The proportion of $A P O E \varepsilon 4$ carriers was high in groups with stronger evidence for AD pathology, and the percentage seemed to increase when applying the more recent criteria (NIA-AA 2011 intermediate AD likelihood 64.5\%, high AD likelihood 72.2\%, IWG-2 prodromal AD 76.9\%, NIA-AA 2018 AD 78.5\%; data not shown).

\section{Disease progression}

Table 3 shows per each set of criteria the estimates for 2 -year change in cognitive and cognitive-functional performance, progression rates to dementia, and hazard ratios (HR) for dementia risk.

\section{IWG-2 criteria}

The changes in NTB scores were modest overall in both IWG-2 groups, and no differences were observed between the groups (Table 3). For CDR-SB, there was more worsening in the prodromal AD group (estimate for change 1.18 points vs. 0.42 points; $p=0.03$ ). This group also had a higher risk of progression to dementia (42.0\% vs. 26.9\%; HR 4.6, 95\% CI 1.6-13.7).

\section{NIA-AA 2011 criteria}

The changes in NTB scores were fairly small in all NIAAA 2011 groups. No statistically significant differences were observed between the reference group SNAP and the other groups, but there was a trend indicating somewhat higher rate of decline in the intermediate and high AD likelihood groups (Table 3). The pattern of decline in these two groups was however similar. Individuals with a high AD likelihood were more likely to progress to dementia than those with SNAP (43.0\% vs. $7.7 \%$; HR 7.4, 95\% CI 1.0-54.7). The risk was similar in the high and intermediate AD likelihood groups.

\section{NIA-AA 2018 criteria}

The changes in NTB were small across all NIA-AA 2018 groups. The AD group showed consistently the highest rate of decline, but the changes did not differ from those of the reference group non-AD pathologic change (Table 3). CDR-SB scores worsened more in the AD group than in the reference group (estimate for change 1.43 points vs. 0.48 points; $p=0.03$ ) and in the Alzheimer's and concomitant suspected non-Alzheimer's pathologic change group (1.43 points vs. 0.43 points; $p=0.01$ ). The AD group was also more likely to progress to dementia than the reference group $(44.1 \%$ vs. $7.7 \%$, HR 9.4, 95\% CI 1.2-72.7) and the Alzheimer's and concomitant suspected non-Alzheimer's pathologic change group (44.1\% vs. $40.0 \%$, HR 2.7 , 95\% CI 1.0-7.0).

\section{Discussion}

In this study, we examined the baseline biomarker profiles of individuals with IWG-1 prodromal AD enrolled in the LipiDiDiet RCT. We found that in the subset of study participants with available centrally analyzed biomarkers (including CSF), most individuals displayed a clear AD biomarker profile and could be classified as having IWG-2 prodromal AD, high AD likelihood (NIAAA 2011), and AD (NIA-AA 2018). Approximately 90\% of those with centrally analyzed CSF had abnormal A $\beta$; most participants also had an $\mathrm{A}+\mathrm{T}+\mathrm{N}+$ profile. In line with studies reporting that the IWG-1 criteria are sensitive and have a decent prognostic accuracy $[8,25]$, our findings suggest that the IWG-1 criteria might reliably capture an early symptomatic AD population-even if they require less comprehensive biomarker evidence 


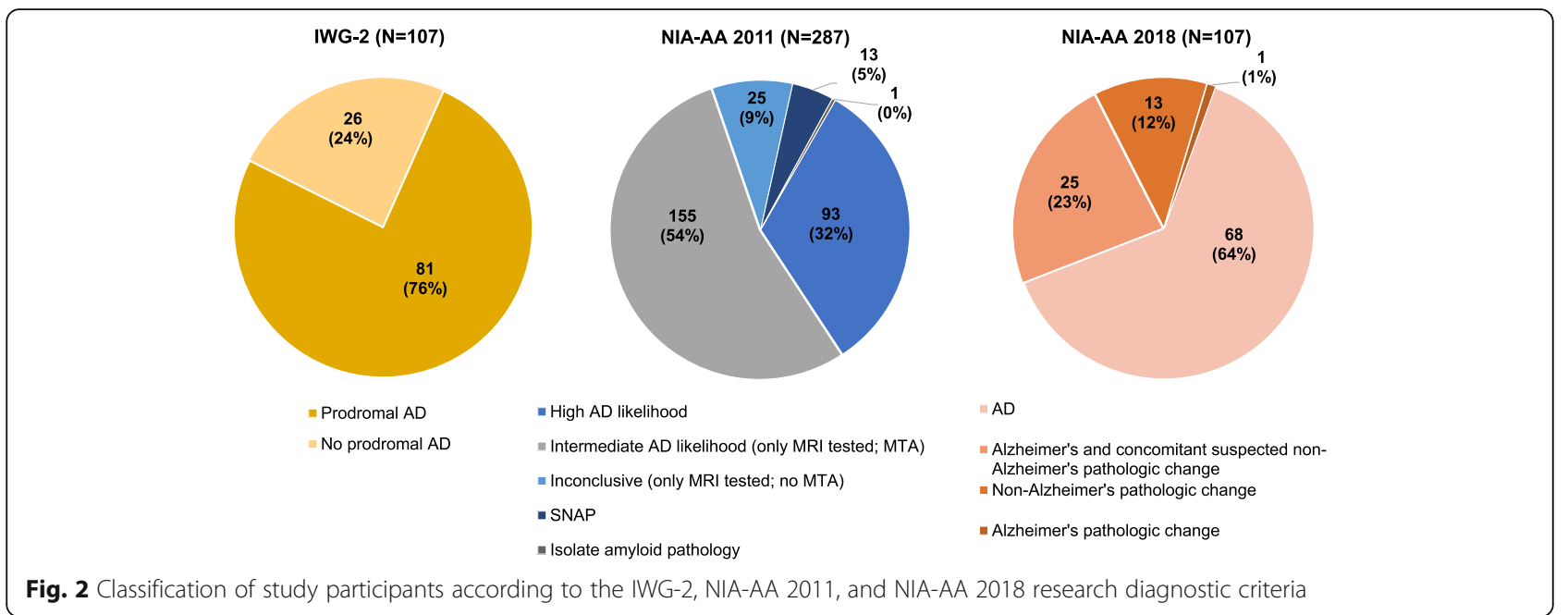

than the more recently proposed criteria. Given that the LipiDiDiet participants-who were primarily memory clinic patients-had amnestic cognitive impairment and often already signs of brain atrophy on MRI, the high prevalence of $A \beta$ positivity and $A+T+N+$ profile was expected. This is because $A \beta$ accumulation is thought to precede neuronal injury, which in turn correlates closely with clinical symptoms [26]. Among the participants whose CSF was analyzed but not all biomarkers were abnormal, a considerable proportion had CSF p-tau within the normal range and an $\mathrm{A}+\mathrm{T}-\mathrm{N}+$ profile, indicating either subthreshold tauopathy or amyloidosis in combination with non-AD pathologies [27-29]. In previous studies, the $\mathrm{A}+\mathrm{T}-\mathrm{N}+$ profile has been uncommon in mild cognitive impairment (MCI), both in memory clinic patients [30, 31] and in more selected cohorts [32, 33]. We observed that even a small adjustment of the p-tau cut-off changed the classification, suggesting that several LipiDiDiet participants with an $\mathrm{A}+\mathrm{T}-\mathrm{N}+$ profile had subthreshold levels of tau. In a recently published study where data-driven methods were applied to determine unbiased cut-off points for tau, researchers identified three different cut-offs instead of a single clear cut-off, resulting in four subgroups of individuals with somewhat different cognitive trajectories [34]. Collectively, these findings and our results underline the issue with using sharp dichotomous biomarker cut-offs to classify individuals.

To further investigate the applicability of the research diagnostic criteria, we examined LipiDiDiet participants' disease progression over 2 years. Cognitive and cognitive-functional decline appeared to be more pronounced in the prodromal AD (IWG-2), high and intermediate AD likelihood (NIA-AA 2011), and AD (NIAAA 2018) groups, but despite some trends, only few statistically significant between-group differences were observed within each set of criteria. The study was likely underpowered to detect these differences. Another explanation for these findings is that the changes, especially in NTB, were modest overall and lower than expected during the first 2 years of the LipiDiDiet trial [17] but closer to the expectation after 3 years [35]. Here, we observed that the changes were fairly small even among those with both abnormal $A \beta$ and neuronal injury markers. With respect to the CDR-SB scores, the increase was more pronounced in the $\mathrm{AD}$ group $(\mathrm{A}+\mathrm{T}+$ $\mathrm{N} \pm$ ) than in the Alzheimer's and concomitant suspected non-Alzheimer's pathologic change group $(\mathrm{A}+\mathrm{T}-\mathrm{N}+)$, potentially supporting the distinction between tau (e.g., p-tau) and other neuronal injury markers. However, we do not know if the between-group differences within each set of criteria would differ at older vs. younger ages, since the LipiDiDiet participants without CSF were older, and older individuals are also more likely to have mixed pathologies [36].

The participants in the LipiDiDiet trial developed dementia at rates proportional to the certainty of underlying $\mathrm{AD}$, and the risk of progression was higher among those with IWG-2 prodromal AD, NIA-AA 2011 high AD likelihood, and NIA-AA 2018 AD. Progression rates were very similar in these groups, highlighting the overlap between the criteria and these categories. Overall, our findings are consistent with previous studies showing that MCI individuals with abnormal $A \beta$ and neuronal injury (IWG-2 prodromal AD, NIA-AA 2011 high $\mathrm{AD}$ likelihood) might have an increased risk of disease progression compared to those with normal/conflicting biomarkers or abnormal $A \beta$ alone $[8,16]$. The few available longitudinal studies investigating the NIA-AA 2018 criteria have also reported an increased risk of decline for the AD profiles, both in MCI $[30,33]$ and among cognitively healthy individuals $[29,37,38]$.

In LipiDiDiet, participant eligibility was often evaluated based on MTA rather than CSF assessment, which 
Table 3 Two-year change from baseline in NTB and CDR-SB scores and progression to dementia according to the IWG-2, NIA-AA 2011, and NIA-AA 2018 criteria

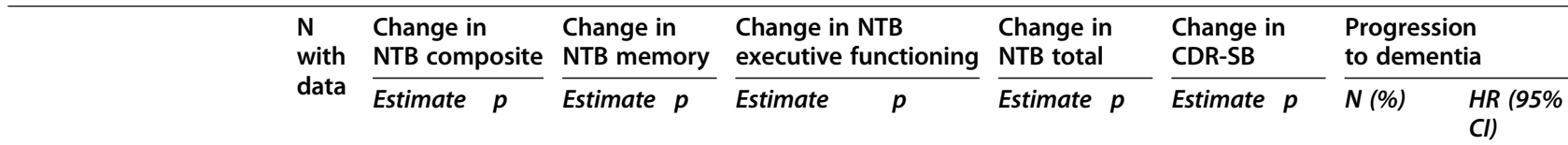

\section{IWG-2}

\begin{tabular}{|c|c|c|c|c|c|c|c|c|c|c|c|c|c|}
\hline No prodromal AD & 24 & -0.018 & Ref & -0.004 & Ref & -0.085 & Ref & 0.048 & Ref & 0.42 & Ref & $\begin{array}{l}7 / 26 \\
(26.9)\end{array}$ & Ref \\
\hline Prodromal AD & 76 & -0.254 & 0.11 & -0.266 & 0.11 & -0.097 & 0.93 & -0.107 & 0.13 & 1.18 & 0.03 & $\begin{array}{l}34 / 81 \\
(42.0)\end{array}$ & $\begin{array}{l}4.6 \\
(1.6-13.7)\end{array}$ \\
\hline
\end{tabular}

\section{NIA-AA 2011}

\begin{tabular}{|c|c|c|c|c|c|c|c|c|c|c|c|c|c|}
\hline SNAP & 13 & 0.081 & Ref & 0.128 & Ref & -0.044 & Ref & 0.057 & Ref & 0.56 & Ref & $1 / 13(7.7)$ & Ref \\
\hline $\begin{array}{l}\text { Inconclusive/ } \\
\text { uninformative }\end{array}$ & 22 & 0.129 & 0.79 & 0.087 & 0.85 & 0.014 & 0.75 & 0.109 & 0.71 & 0.21 & 0.51 & $\begin{array}{l}4 / 25 \\
(16.0)\end{array}$ & $\begin{array}{l}2.0 \\
(0.2-18.7)\end{array}$ \\
\hline $\begin{array}{l}\text { Intermediate AD } \\
\text { likelihood }\end{array}$ & 135 & -0.192 & 0.07 & -0.209 & 0.06 & -0.144 & 0.50 & -0.169 & 0.05 & 1.29 & 0.08 & $\begin{array}{l}64 / 155 \\
(41.3)\end{array}$ & $\begin{array}{l}6.8 \\
(0.9-50.0)\end{array}$ \\
\hline High AD likelihood & 86 & -0.195 & 0.08 & -0.186 & 0.08 & -0.083 & 0.80 & -0.084 & 0.23 & 1.08 & 0.23 & $\begin{array}{l}40 / 93 \\
(43.0)\end{array}$ & $\begin{array}{l}7.4 \\
(1.0-54.7)\end{array}$ \\
\hline \multicolumn{14}{|l|}{ VIA-AA 2018} \\
\hline $\begin{array}{l}\text { Non-Alzheimer's } \\
\text { pathologic change }\end{array}$ & 13 & 0.057 & Ref & 0.049 & Ref & -0.088 & Ref & 0.038 & Ref & 0.48 & Ref & $1 / 13(7.7)$ & Ref \\
\hline $\begin{array}{l}\text { Alzheimer's and } \\
\text { concomitant suspected } \\
\text { non-Alzheimer's } \\
\text { pathologic change }\end{array}$ & 22 & -0.154 & 0.31 & -0.125 & 0.45 & -0.112 & 0.91 & 0.029 & 0.96 & 0.43 & 0.91 & $\begin{array}{l}10 / 25 \\
(40.0)\end{array}$ & $\begin{array}{l}3.5 \\
(0.4-29.4)\end{array}$ \\
\hline$A D$ & 64 & -0.274 & 0.08 & -0.299 & 0.09 & -0.087 & 0.99 & -0.134 & 0.20 & 1.43 & 0.03 & $\begin{array}{l}30 / 68 \\
(44.1)\end{array}$ & $\begin{array}{l}9.4 \\
(1.2-72.7)\end{array}$ \\
\hline
\end{tabular}

Estimates for change in NTB and CDR-SB scores are least-squares means for change from baseline over 2 years within each group. Negative values indicate decline over time, except for CDR-SB where positive values indicate decline. $p$-values are shown for the difference in the least-squares means over 2 years between each group and the respective reference group. Numbers of participants are as per modified intention-to-treat (mITT) analysis. CDR-SB data were missing for nine participants (IWG-2, NIA-AA 2018) and 32 participants (NIA-AA 2011). Isolate amyloid pathology/Alzheimer's pathologic change was excluded from the analyses $(N=1)$

Abbreviations: $A D$, Alzheimer's disease; $C D R-S B$, Clinical Dementia Rating-Sum of Boxes; IWG, International Working Group; NIA-AA, National Institute on AgingAlzheimer's Association; NTB, Neuropsychological Test Battery; SNAP, suspected non-AD pathology

is why a large group of individuals were classified in the NIA-AA 2011 intermediate AD likelihood group. Notably, we observed that the rate of disease progression was consistently similar in this group and in the high AD likelihood group. This is an encouraging result, given that the assessment of only one biomarker (usually MTA on widely available MRI) is a common scenario. Methods for measuring $A \beta$ are currently invasive (lumbar puncture) and costly (PET), limiting their application in routine clinical practice and in RCTs conducted in diverse settings. While the IWG- 2 and NIA-AA criteria might have a higher specificity $[8,25]$, the IWG-1 criteria could be preferred in some situations, yet further investigation is needed to identify the optimal set of criteria for participant recruitment to prevention RCT. Ongoing validation studies will also show if plasma biomarkers for p-tau and $A \beta$ can help streamline recruitment for future prevention RCTs [39-41].

One advantage of the IWG-1 criteria over the other research criteria is related to the efficiency of recruitment. The use of more restrictive criteria decreases the number of eligible individuals, as shown also in our study. The choice of criteria may also have implications for the representativeness of the study population. We found that the participants who underwent lumbar puncture for CSF analysis were somewhat younger and had a better cognitive-functional performance than those who did not. Reasons for this are unclear, but older participants might have had more often contraindications for the procedure. In one previous memory clinic study, contraindications were indeed often observed among patients who did not undergo routine lumbar puncture [42]. This study did not find any age difference, but the latter group did have a lower MMSE [42]. In LipiDiDiet, a possible explanation for the observed differences is that the study populations could have been slightly different at different sites, depending on local circumstances. Sites where MRI was the preferred method might have had older patients with poorer cognitive-functional performance. Little is known 
about the impact of selection criteria on participant characteristics in prodromal AD RCTs, but strict biomarker criteria could limit the study population representativeness through exclusion of many older individuals who often have mixed pathologies [36]. Whenever possible considering the nature of the intervention, including such individuals in RCTs is encouraged as they form a large group of potential intervention end users.

\section{Limitations}

The study population was largely Caucasian and a homogenous group of RCT participants recruited primarily from memory clinics. Our findings may thus not be fully generalizable to other populations or settings, and further investigation will be needed in ethnically and geographically more diverse cohorts, ideally with a longer timeframe to assess disease progression. In the LipiDiDiet RCT, the IWG- 1 criteria for prodromal AD were used to recruit participants, and the eligibility criteria included evidence for underlying $\mathrm{AD}$ pathology based on either imaging or CSF markers (i.e., participants were not required to have both assessments). Therefore, CSF was not available for the whole study population, and the classification according to certain criteria (IWG-2, NIA-AA 2018) was possible only in a subset of participants. Furthermore, as the sample was overall small, and only few participants were classified in the groups reflecting lower certainty of $\mathrm{AD}$ (e.g., low $\mathrm{AD}$ likelihood, SNAP), we lacked statistical power to detect between-group differences in the longitudinal analyses of disease progression. The smaller than expected cognitive changes over a rather short follow-up period of 2 years could have also affected our results. LipiDiDiet extension studies will shed light on the longer-term cognitive and cognitive-functional trajectories and prognosis in prodromal $\mathrm{AD}$, which could improve the operationalization of the research diagnostic criteria. Another limitation of our study is that the $A \beta$ and tau assessment was based only on CSF. CSF and PET reflect different aspects of pathology [43] and incorporating PET could have affected the classification. Nevertheless, $A \beta$ status was determined based on both $A \beta 42$ and $A \beta 42 / 40$ ratio, which is an advantage as the latter parameter is potentially a more accurate measure of $A \beta$ pathology [44]. Another major strength of our study was the possibility to investigate the research diagnostic criteria in a real-life RCT setting. This is because all biomarkers were centrally analyzed, and the sampling and assessment protocols were standardized. In multicenter studies, variability introduced by different laboratory procedures and cutoffs is a common challenge. Cognitive testing was also standardized in our study.

\section{Conclusions}

Assessing the applicability of different participant selection criteria is crucial to inform the design and recruitment of future RCTs. Criteria should be restrictive enough to ensure inclusion of the right population with potential to respond to the interventions, yet feasibility and differences in clinical practices are important considerations. Our findings indicate that the IWG-1 criteria might reliably identify individuals with $\mathrm{AD}$ pathology, supporting the use of these criteria in certain prevention RCTs targeting pre-dementia stages. Firstly, while complex assessments like CSF and PET are currently necessary to verify biomarker status in $A \beta$ - or tau-targeting RCTs, more pragmatic and easily applicable criteria could be preferred due to feasibility in trials investigating, e.g., non-pharmacological lifestyle-based interventions. These interventions do not necessarily target any specific pathology but exert their effects through multiple mechanisms of action. Secondly, it is important that prevention strategies are tested also in settings where possibilities for comprehensive biomarker testing are limited, and in these situations, pragmatic criteria like the IWG-1 could potentially be considered. At the moment, several multidomain prevention RCTs are being planned and conducted worldwide, many of them within the World Wide FINGERS (WW-FINGERS) network [45].

\section{Abbreviations \\ $A \beta$ : $\beta$-amyloid; $A \beta 42$ : $\beta$-amyloid 1-42; $A \beta 42 / 40$ : Ratio of $\beta$-amyloid 1-42 and $\beta$-amyloid 1-40; AD: Alzheimer's disease; APOE: Apolipoprotein E; A/T/N: Amyloid/tau/neuronal injury classification; CDR-SB: Clinical Dementia Rating-Sum of Boxes; CERAD: Consortium to Establish a Registry for Alzheimer's Disease; CSF: Cerebrospinal fluid; DSM-IV: Diagnostic and Statistical Manual of Mental Disorders 4th edition; FDG-PET: Fluoro-deoxy- glucose-positron emission tomography; IWG: International Working Group; MCI: Mild cognitive impairment; mlTT: Modified intention-to-treat; MMSE: Mini-Mental State Examination; MRI: Magnetic resonance imaging; MTA: Medial temporal lobe atrophy; NIA-AA: National Institute on Aging- Alzheimer's Association; NINCDS-ADRDA: National Institute of Neurological and Communicative Disorders and Stroke-Alzheimer's Disease and Related Disorders Association; NTB: Neuropsychological Test Battery; PET: Positron emission tomography; p-tau: Phosphorylated tau protein (at threonine 181); RCT: Randomized controlled trial; SNAP: Suspected non-AD pathology; t- tau: Total tau protein; WMS-r: Wechsler Memory Scale-revised; \\ WW-FINGERS: World Wide FINGERS}

\section{Acknowledgements}

We sincerely thank all participants enrolled in the study and their families. Members of the LipiDiDiet clinical study group: Hilkka Soininen, Ilona Hallikainen, Merja Hallikainen, Seppo Helisalmi, Tarja Lappalainen, Yawu Liu, Teemu Paajanen (University of Eastern Finland, Kuopio, Finland); Miia Kivipelto, Alina Solomon, Lars-Olof Wahlund, Yvonne Freund-Levi, Göran Hagman (Karolinska University Hospital, Stockholm, Sweden); Kaj Blennow (University of Gothenburg, Gothenburg, Sweden); Tobias Hartmann, Klaus Fassbender, Matthias Riemenschneider, Marcus OW Grimm, Aline KleesRollmann, Maxine Luley, Epameinondas Lyros, Robert Schomburg, Daniela Ramelli, Jennifer Kennel (Saarland University, Homburg, Germany); Lutz Frölich, Lucrezia Hausner (Central Institute of Mental Health, Mannheim, Germany); Christoph Laske, Thomas Leyhe, Christian Mychajliw, Niklas Koehler (University Hospital of Tübingen, Tübingen, Germany); Stephan Schiekofer, Hans Klünemann (University Hospital Regensburg, Regensburg, Germany); Johannes Schröder (Heidelberg University Hospital, Heidelberg, Germany); 
Dieter Lütjohann (Universitätsklinikum Bonn, Bonn, Germany); Pieter Jelle Visser, Philip Scheltens, Ineke van Rossum, Nienke Scheltens, Daniela Bertens, Mara ten Kate, Frederik Barkhof, Silvia Ingala (VU University Medical Center, Amsterdam, the Netherlands); Johanna ML Henselmans (Antonius Hospital, Woerden, the Netherlands); Gerwin Roks (St. Elisabeth Hospital, Tilburg, the Netherlands); Anneke MJ van Hees, Floor M van Oudenhoven (Danone Nutricia Research, Utrecht, the Netherlands); Suzanne B Hendrix, Noel Ellison (Pentara Corporation, Millcreek, UT, USA).

\section{Authors' contributions}

Study concept and design: AR, AS, HS, PJV, KB, TH, and MK. Analysis and interpretation of data: AR and AS. Data acquisition: AS, HS, PJV, KB, and MK. Drafting/revising the manuscript for content: AR, AS, HS, PJV, KB, TH, and MK. All authors read and approved the final manuscript. AR and AS contributed equally as first authors.

\section{Funding}

The research leading to these results was mainly funded by the European Commission under the 7th framework programme of the European Union [grant agreement no. 211696]; additional funding for this study was provided by the EU Joint Programme - Neurodegenerative Disease Research (JPND) MIND-AD grant to MK, HS, TH; EU Joint Programme - Neurodegenerative Disease Research (JPND) EURO-FINGERS grant to MK, TH; Kuopio University Hospital, Finland (EVO/NTR grant; HS); Alzheimerfonden Sweden; Swedish Research Council; Stockholm City Council (ALF grant); Center for Innovative Medicine (CIMED) at Karolinska Institute, Sweden; Stiftelsen Stockholms sjukhem, Sweden; European Research Council [grant 804371]; and Academy of Finland grants [317465, 287490]. KB is supported by Alzheimerfonden Sweden (\#AF-742881), Hjärnfonden Sweden (\#FO2017-0243), and the Swedish state under the agreement between the Swedish government and the County Councils, the ALF-agreement (\#ALFGBG-715986). AR was supported by Finnish Cultural Foundation, Finnish Brain Foundation, Maire Taponen Foundation, and Emil Aaltonen Foundation.

The European Commission had no role in the study design, data collection analysis, and interpretation; writing of the report; or the decision to submit for publication. Danone Nutricia Research had no role in the study design, data collection, and the decision to submit for publication, and as part of the LipiDiDiet consortium was involved in data analysis and writing of the current report. All decisions taken on design, analysis, interpretation, and decision to submit for publication were made by the LipiDiDiet Trial Steering Committee, excluding Danone Nutricia Research. HS, AS, PJV, KB, MK, and TH had full access to all the data in the study. The corresponding author had final responsibility for the decision to submit for publication.

\section{Availability of data and materials}

LipiDiDiet is an ongoing study. The LipiDiDiet consortium is open to all requests from external researchers for data collected in the trial. The study protocol is publicly available online at http://lipididiet.eu/fileadmin/lipididiet/ publications/LipiDiDietStudyProtocol.pdf. Requesters will be asked to submit a study protocol, including the research question, planned analysis, and data required. The LipiDiDiet Trial Steering Committee will evaluate this plan (i.e., relevance of the research question, suitability of the data, quality of the proposed analysis, planned or ongoing LipiDiDiet analysis, and other matters) on a case-by-case basis and provide the data or reject the request. Shared data will encompass the data dictionary and de-identified participant data only. Any analysis will be conducted in collaboration with and on behalf of the LipiDiDiet consortium. Access is subject to the LipiDiDiet legal framework. An access agreement will be prepared and signed by both parties.

\section{Declarations}

\section{Ethics approval and consent to participate}

Ethical approval was granted by local ethics committees at each study site, and written informed consent was obtained from all participants and study partners. Approval was obtained from the following committees: Ethics committee of the Hospital District of Northern Savo in Finland (PohjoisSavon sairaanhoitopiirin tutkimuseettinen toimikunta), the Stockholm Regional Ethical Review Board in Sweden (Regionala etikprövningsnämnden i Stockholm), the Medical Ethical Committee of the VU University Medical Center Amsterdam in the Netherlands (Medisch Ethische Toetsingscommissie $\mathrm{VU}$ medisch centrum), and the ethical committees of University of
Regensburg (Fakultät Medizin Ethikkommission Universität Regensburg), Eberhards-Karls-University of Tübingen and Tübingen University Hospital Clinic (Ethik-Kommission an der Medizinischen Fakultät der Eberhard-KarlsUniversität und am Universitätsklinikum Tübingen), Heidelberg University (Ethikkommission Medizinische Fakultät Heidelberg), the Medical faculty in Mannheim (Medizinische Fakultät Mannheim Medizinische Ethik-Kommission II), and Saarland University (Ethik-Kommission Ärtzekammer des Saarlandes) in Germany.

\section{Consent for publication}

Not applicable.

\section{Competing interests}

The LipiDiDiet consortium received funding by Danone Nutricia Research for the intervention period from 25 to 96 months, and the consortium distributed the funding to their members to conduct the trial and analysis. HS reports personal fees from ACl and MERCK (advisor), outside the submitted work. KB has served as a consultant, at advisory boards, or at data monitoring committees for Abcam, Axon, Biogen, JOMDD/Shimadzu, Julius Clinical, Lilly, MagQu, Novartis, Roche Diagnostics, and Siemens Healthineers and is a co-founder of Brain Biomarker Solutions in Gothenburg AB (BBS), which is a part of the GU Ventures Incubator Program (disclosures not relevant for the submitted work). AR, AS, PJV, TH, and MK report no competing interests.

\section{Author details}

'Department of Neurology, Institute of Clinical Medicine, University of Eastern Finland, Kuopio, Finland. ${ }^{2}$ Division of Clinical Geriatrics, Centre for Alzheimer Research, Department of Neurobiology, Care Sciences and Society, Karolinska Institutet, Stockholm, Sweden. ${ }^{3}$ Theme Aging, Karolinska University Hospital, Stockholm, Sweden. ${ }^{4}$ Neurocenter, Department of Neurology, Kuopio University Hospital, Kuopio, Finland. ${ }^{5}$ Department of Psychiatry and Neuropsychology, Alzheimer Centre Limburg, University of Maastricht, Maastricht, Netherlands. 'Department of Neurology, Alzheimer Centre, Amsterdam Neuroscience, VU University Medical Centre, Amsterdam, Netherlands. 'Department of Psychiatry and Neurochemistry, Institute of Neuroscience and Physiology, The Sahlgrenska Academy at University of Gothenburg, Mölndal, Sweden. ${ }^{8}$ Clinical Neurochemistry Laboratory, Sahlgrenska University Hospital, Mölndal, Sweden. ${ }^{9}$ Deutsches Institut für Demenz Prävention (DIDP), Medical Faculty, and Department of Experimental Neurology, Saarland University, Homburg, Germany. ${ }^{10}$ Institute of Public Health and Clinical Nutrition, University of Eastern Finland, Kuopio, Finland.

${ }^{11}$ Ageing Epidemiology Research Unit, School of Public Health, Imperial College London, London, UK.

\section{Received: 12 November 2020 Accepted: 23 February 2021}

Published online: 25 March 2021

\section{References}

1. Dubois B, Feldman HH, Jacova C, DeKosky ST, Barberger-Gateau P, Cummings J, et al. Research criteria for the diagnosis of Alzheimer's disease: revising the NINCDS-ADRDA criteria. Lancet Neurol. 2007;6:734-46.

2. Dubois B, Feldman HH, Jacova C, Cummings JL, Dekosky ST, BarbergerGateau P, et al. Revising the definition of Alzheimer's disease: a new lexicon. Lancet Neurol. 2010;9:1118-27.

3. Albert MS, DeKosky ST, Dickson D, Dubois B, Feldman HH, Fox NC, et al. The diagnosis of mild cognitive impairment due to Alzheimer's disease: recommendations from the National Institute on Aging-Alzheimer's Association workgroups on diagnostic guidelines for Alzheimer's disease. Alzheimers Dement. 2011;7:270-9.

4. Sperling RA, Aisen PS, Beckett LA, Bennett DA, Craft S, Fagan AM, et al. Toward defining the preclinical stages of Alzheimer's disease: recommendations from the National Institute on Aging-Alzheimer's Association workgroups on diagnostic guidelines for Alzheimer's disease. Alzheimers Dement. 2011;7:280-92.

5. Dubois $\mathrm{B}$, Feldman $\mathrm{HH}$, Jacova $\mathrm{C}$, Hampel $\mathrm{H}$, Molinuevo $\mathrm{JL}$, Blennow $\mathrm{K}$, et al. Advancing research diagnostic criteria for Alzheimer's disease: the IWG-2 criteria. Lancet Neurol. 2014;13:614-29.

6. Dubois B, Hampel H, Feldman HH, Scheltens P, Aisen P, Andrieu S, et al. Preclinical Alzheimer's disease: definition, natural history, and diagnostic criteria. Alzheimers Dement. 2016;12:292-323. 
7. Jack CR, Bennett DA, Blennow K, Carrillo MC, Dunn B, Haeberlein SB, et al. NIA-AA research framework: toward a biological definition of Alzheimer's disease. Alzheimers Dement. 2018;14:535-62.

8. Vos SJB, Verhey F, Frölich L, Kornhuber J, Wiltfang J, Maier W, et al. Prevalence and prognosis of Alzheimer's disease at the mild cognitive impairment stage. Brain. 2015;138:1327-38.

9. Siemers ER, Sundell KL, Carlson C, Case M, Sethuraman G, Liu-Seifert H, et al. Phase 3 solanezumab trials: secondary outcomes in mild Alzheimer's disease patients. Alzheimers Dement. 2016;12:110-20.

10. Salloway S, Sperling R, Fox NC, Blennow K, Klunk W, Raskind M, et al. Two phase 3 trials of bapineuzumab in mild-to-moderate Alzheimer's disease. N Engl J Med. 2014;370:322-33.

11. Grill JD, Nuño MM, Gillen DL. Which MCl patients should be included in prodromal Alzheimer disease clinical trials? Alzheimer Dis Assoc Disord. 2019;33:104-12.

12. Schneider LS, Kennedy RE, Cutter GR. Requiring an amyloid-beta1-42 biomarker for prodromal Alzheimer's disease or mild cognitive impairment does not lead to more efficient clinical trials. Alzheimers Dement. 2010;6: 367-77.

13. Holland D, McEvoy LK, Desikan RS, Dale AM. Enrichment and stratification for predementia Alzheimer disease clinical trials. PLoS One. 2012;7:e47739.

14. Insel PS, Mattsson N, Mackin RS, Kornak J, Nosheny R, Tosun-Turgut D, et al. Biomarkers and cognitive endpoints to optimize trials in Alzheimer's disease. Ann Clin Transl Neurol. 2015;2:534-47.

15. Wolz R, Schwarz AJ, Gray KR, Yu P, Hill DLG. Enrichment of clinical trials in $\mathrm{MCl}$ due to $\mathrm{AD}$ using markers of amyloid and neurodegeneration. Neurology. 2016;87:1235-41.

16. Bertens D, Tijms BM, Vermunt L, Prins ND, Scheltens P, Visser PJ. The effect of diagnostic criteria on outcome measures in preclinical and prodromal Alzheimer's disease: implications for trial design. Alzheimers Dement Trans Res Clin Interv. 2017;3:513-23.

17. Soininen $H$, Solomon A, Visser PJ, Hendrix SB, Blennow K, Kivipelto M, et al. 24-month intervention with a specific multinutrient in people with prodromal Alzheimer's disease (LipiDiDiet): a randomised, double-blind, controlled trial. Lancet Neurol. 2017;16:965-75.

18. de Wilde MC, Vellas B, Girault E, Yavuz AC, Sijben JW. Lower brain and blood nutrient status in Alzheimer's disease: results from meta-analyses. Alzheimers Dement Transl Res Clin Interv. 2017:3:416-31.

19. Scheltens P, Kamphuis PJGH, Verhey FRJ, Olde Rikkert MGM, Wurtman RJ, Wilkinson D, et al. Efficacy of a medical food in mild Alzheimer's disease: a randomized, controlled trial. Alzheimers Dement. 2010;6:1-10 e1.

20. Scheltens P, Twisk JWR, Blesa R, Scarpini E, Von Arnim CAF, Bongers A, et al. Efficacy of souvenaid in mild Alzheimer's disease: results from a randomized, controlled trial. J Alzheimers Dis. 2012;31:225-36.

21. American Psychiatric Association. Diagnostic and statistical manual of mental disorders. 4th ed. Washington, DC: American Psychiatric Association, 1994.

22. McKhann G, Drachman D, Folstein M, Katzman R, Price D, Stadlan EM Clinical diagnosis of Alzheimer's disease: report of the NINCDS-ADRDA Work Group under the auspices of Department of Health and Human Services Task Force on Alzheimer's Disease. Neurology. 1984;34:939-44.

23. Jack CR, Bennett DA, Blennow K, Carrillo MC, Feldman HH, Frisoni GB, et al. $\mathrm{A} / \mathrm{T} / \mathrm{N}$ : an unbiased descriptive classification scheme for Alzheimer disease biomarkers. Neurology. 2016;87:539-47.

24. Jack CR, Knopman DS, Weigand SD, Wiste HJ, Vemuri P, Lowe V, et al. An operational approach to National Institute on Aging-Alzheimer's Association criteria for preclinical Alzheimer disease. Ann Neurol. 2012;71:765-75.

25. Prestia A, Caroli A, Wade SK, van der Flier WM, Ossenkoppele R, Van Berckel $B$, et al. Prediction of AD dementia by biomarkers following the NIA-AA and IWG diagnostic criteria in $\mathrm{MCl}$ patients from three European memory clinics. Alzheimers Dement. 2015;11:1191-201.

26. Jack CR, Knopman DS, Jagust WJ, Petersen RC, Weiner MW, Aisen PS, et al. Tracking pathophysiological processes in Alzheimer's disease: an updated hypothetical model of dynamic biomarkers. Lancet Neurol. 2013;12:207-16.

27. Nelson PT, Dickson DW, Trojanowski JQ, Jack CR, Boyle PA, Arfanakis K, et al. Limbic-predominant age-related TDP-43 encephalopathy (LATE): consensus working group report. Brain. 2019;142:1503-27.

28. Botha H, Mantyh WG, Graff-Radford J, Machulda MM, Przybelski SA, Wiste $\mathrm{HJ}$, et al. Tau-negative amnestic dementia masquerading as Alzheimer disease dementia. Neurology. 2018;90:e940-6.

29. Jack CR, Wiste HJ, Therneau TM, Weigand SD, Knopman DS, Mielke MM, et al. Associations of amyloid, tau, and neurodegeneration biomarker profiles with rates of memory decline among individuals without dementia. JAMA. 2019:321:2316-25.

30. Altomare D, De Wilde A, Ossenkoppele R, Pelkmans W, Bouwman F, Groot C, et al. Applying the ATN scheme in a memory clinic population: the ABIDE project. Neurology. 2019;93:e1635-46.

31. Dodich A, Mendes A, Assal F, Chicherio C, Rakotomiaramanana B, Andryszak $\mathrm{P}$, et al. The A/T/N model applied through imaging biomarkers in a memory clinic. Eur J Nucl Med Mol Imaging. 2020;47:247-55.

32. Burnham SC, Coloma PM, Li Q-X, Collins S, Savage G, Laws S, et al. Application of the NIA-AA research framework: towards a biological definition of Alzheimer's disease using cerebrospinal fluid biomarkers in the AIBL study. J Prev Alzheimers Dis. 2019;6:248-55

33. Ekman U, Ferreira D, Westman E. The A/T/N biomarker scheme and patterns of brain atrophy assessed in mild cognitive impairment. Sci Rep. 2018;8: 8431

34. Duits FH, Wesenhagen KEJ, Ekblad L, Wolters E, Willemse EAJ, Scheltens P, et al. Four subgroups based on tau levels in Alzheimer's disease observed in two independent cohorts. Alzheimers Res Ther. 2021;13:2.

35. Soininen $H$, Solomon A, Visser PJ, Hendrix SB, Blennow K, Kivipelto M, et al. 36-month LipiDiDiet multinutrient clinical trial in prodromal Alzheimer's disease. Alzheimers Dement. 2021;17:29-40.

36. Boyle PA, Yu L, Wilson RS, Leurgans SE, Schneider JA, Bennett DA. Personspecific contribution of neuropathologies to cognitive loss in old age. Ann Neurol. 2018:83:74-83.

37. Soldan A, Pettigrew C, Fagan AM, Schindler SE, Moghekar A, Fowler C, et al. ATN profiles among cognitively normal individuals and longitudinal cognitive outcomes. Neurology. 2019;92:e1567-79.

38. Ebenau JL, Timmers T, Wesselman LMP, Verberk IMW, Verfaillie SCJ, Slot RER, et al. ATN classification and clinical progression in subjective cognitive decline: the SCIENCe project. Neurology. 2020;95:e46-58.

39. Nakamura A, Kaneko N, Villemagne VL, Kato T, Doecke J, Doré V, et al. High performance plasma amyloid- $\beta$ biomarkers for Alzheimer's disease. Nature. 2018:554:249-54.

40. Karikari TK, Pascoal TA, Ashton NJ, Janelidze S, Benedet AL, Rodriguez JL, et al. Blood phosphorylated tau 181 as a biomarker for Alzheimer's disease: a diagnostic performance and prediction modelling study using data from four prospective cohorts. Lancet Neurol. 2020;19:422-33.

41. Palmqvist S, Janelidze S, Quiroz YT, Zetterberg H, Lopera F, Stomrud E, et al. Discriminative accuracy of plasma phospho-tau217 for Alzheimer disease vs other neurodegenerative disorders. JAMA. 2020;324:772-81.

42. Duits FH, Prins ND, Lemstra AW, Pijnenburg YAL, Bouwman FH, Teunissen CE, et al. Diagnostic impact of CSF biomarkers for Alzheimer's disease in a tertiary memory clinic. Alzheimers Dement. 2015;11:523-32.

43. Mattsson N, Insel PS, Donohue M, Landau S, Jagust WJ, Shaw LM, et al. Independent information from cerebrospinal fluid amyloid- $\beta$ and florbetapir imaging in Alzheimer's disease. Brain. 2015;138:772-83.

44. Janelidze $S$, Zetterberg $H$, Mattsson N, Palmqvist S, Vanderstichele $H$, Lindberg $O$, et al. CSF $A \beta 42 / A \beta 40$ and $A \beta 42 / A \beta 38$ ratios: better diagnostic markers of Alzheimer disease. Ann Clin Transl Neurol. 2016;3:154-65.

45. Kivipelto M, Mangialasche F, Snyder HM, Allegri R, Andrieu S, Arai H, et al. World-Wide FINGERS Network: a global approach to risk reduction and prevention of dementia. Alzheimers Dement. 2020;16:1078-94.

\section{Publisher's Note}

Springer Nature remains neutral with regard to jurisdictional claims in published maps and institutional affiliations.

Ready to submit your research? Choose BMC and benefit from:

- fast, convenient online submission

- thorough peer review by experienced researchers in your field

- rapid publication on acceptance

- support for research data, including large and complex data types

- gold Open Access which fosters wider collaboration and increased citations

- maximum visibility for your research: over $100 \mathrm{M}$ website views per year

At $\mathrm{BMC}$, research is always in progress.

Learn more biomedcentral.com/submission 